\title{
Use of casts in the necropsy diagnosis of fetal congenital heart disease
}

Andrew C Cook, Nuala L K Fagg, Lindsey D Allan

\begin{abstract}
Objective-To evaluate a casting technique in the interpretation of fetal cardiac anatomy.

Design-In 32 fetuses, the echocardiographic and cast features were compared and correlated.
\end{abstract}

Patients-Three normal fetal heart specimens from spontaneous abortuses and 32 specimens from spontaneous or induced abortions with congenital heart malformation.

Results-There was close correlation between the echocardiographic and anatomical features in 32 abnormal fetuses studied. In some, additional features of diagnosis could be displayed on the cast and the relative sizes of the cardiac structures could be appreciated and defined.

Conclusions-With increasing echocardiographic detection of congenital heart disease in early prenatal life, an increasing number of fetal heart specimens of small size are dissected for pathological confirmation. The use of silicone rubber casts to reproduce the internal anatomy proved a useful addition to dissection, providing a three dimensional model of the cardiac defect.

(Br Heart J 1992;68:481-4)

Fetal echocardiography has become established as a reliable technique for the evaluation of the normal and abnormal fetal heart. ${ }^{1-3}$ Echocardiographic and anatomical correlation has formed an important part of the learning experience in this new field. ${ }^{34}$ The formation of casts of the cardiovascular system has been described since the 16 th century. Leonardo da Vinci referred to the use of wax for casting the cavity of a bull's heart in his notebooks and a number of other materials have been used. Thompsett achieved very accurate representation of the cardiac chambers by using resins and then dissolving the surrounding tissues in acid. ${ }^{5}$ As this involves destruction of the anatomical specimen, the technique has not been generally applied to clinical practice. More recently, silastic polymers mixed with
Department of Pathology, Guy's Hospital London A C Cook N L K Fagg

Department of Fetal Cardiology, Guy's Hospital, London L D Allan

Correspondence to: Professor L D Allan, Department of Fetal Cardiology, 15th Floor Guy's Tower, London SE1 9RT.

Accepted for publication 9 June 1992.
Data on cases

\begin{tabular}{|c|c|c|c|}
\hline Case & Echocardiographic diagnosis & $\begin{array}{l}\text { Gestation } \\
\text { (weeks) }\end{array}$ & Additional cast finding \\
\hline 1 & Distal arch hypoplasia & 20 & Extent of hypoplasia defined \\
\hline 2 & HLHS, arch hypoplasia & 20 & $1.5 \mathrm{~mm}$ VSD \\
\hline 3 & Critical AS & 19 & Coarctation \\
\hline 4 & Critical AS & 20 & Arch hypoplasia, bicuspid AoV \\
\hline 5 & Critical AS & 23 & Unicuspid AoV, coarctation \\
\hline 6 & Mitral atresia, DORV & 23 & Size of VSD and LV defined \\
\hline 7 & Critical AS & 18 & Imperforate AoV, intact atrial septum \\
\hline 8 & HLHS & 22 & Unicuspid AoV, coarctation \\
\hline 9 & VSD & 23 & Coarctation, isthmal hypoplasia \\
\hline 10 & Critical AS & 21 & Isthmal hypoplasia \\
\hline 11 & Critical AS, arch hypoplasia & 18 & Extent of hypoplasia defined \\
\hline 12 & Aortic atresia, Corr TGA & 20 & Coarctation \\
\hline 13 & HLHS & 18 & Aorta and LV size defined \\
\hline 14 & HLHS & 19 & Arch hypoplasia, coarctation \\
\hline 15 & Severe coarctation & 20 & $1 \mathrm{~mm}$ subaortic VSD \\
\hline 16 & HLHS & 19 & Coarctation, arch size defined \\
\hline 17 & HLHS & 22 & Tubular aortic hypoplasia \\
\hline 18 & $\mathrm{RV}>\mathrm{LV}$, trisomy 13 & 20 & Normal arch \\
\hline 19 & HLHS & 21 & Coarctation \\
\hline 20 & Normal heart, hydrops & 26 & Isthmal hypoplasia \\
\hline 21 & HLHS, VSD & 20 & Two VSDs, coarctation, aortic override \\
\hline 22 & Critical AS & 20 & LV size defined \\
\hline 23 & Pulmonary atresia, IVS & 23 & Sinusoid identified \\
\hline 24 & HLHS, LAI, azygos vein & 18 & Azygos defined \\
\hline 25 & Critical AS & 24 & LV size defined \\
\hline 26 & HLHS & 19 & Coarctation. $2 \mathrm{~mm}$ subaortic VSD \\
\hline 27 & AVSD, DORV, LAI & 19 & Azygos vein defined \\
\hline 28 & Corr TGA, aortic atresia & 23 & Coarctation \\
\hline 29 & Corr 'TGA, Ebstein's & 22 & Arch hypoplasia \\
\hline 30 & Corr 'TGA, Ebstein's & 22 & Severe coarctation \\
\hline 31 & TGA, VSD & 19 & Corr TGA, severe arch hypoplasia \\
\hline 32 & Coarctation & 19 & Isthmal stenosis \\
\hline
\end{tabular}

HLHS, hypoplastic left heart syndrome; VSD, ventricular septal defect; AS, aortic stenosis; AoV, aortic valve; DORV, double outlet right ventricle; LV, left ventricle; Corr TGA, corrected transposition of the great arteries; RV, right ventricle; IVS, intact vent ricular septum; LAI, left atrial isomerism; ASVD, atrioventricular septal defect. 


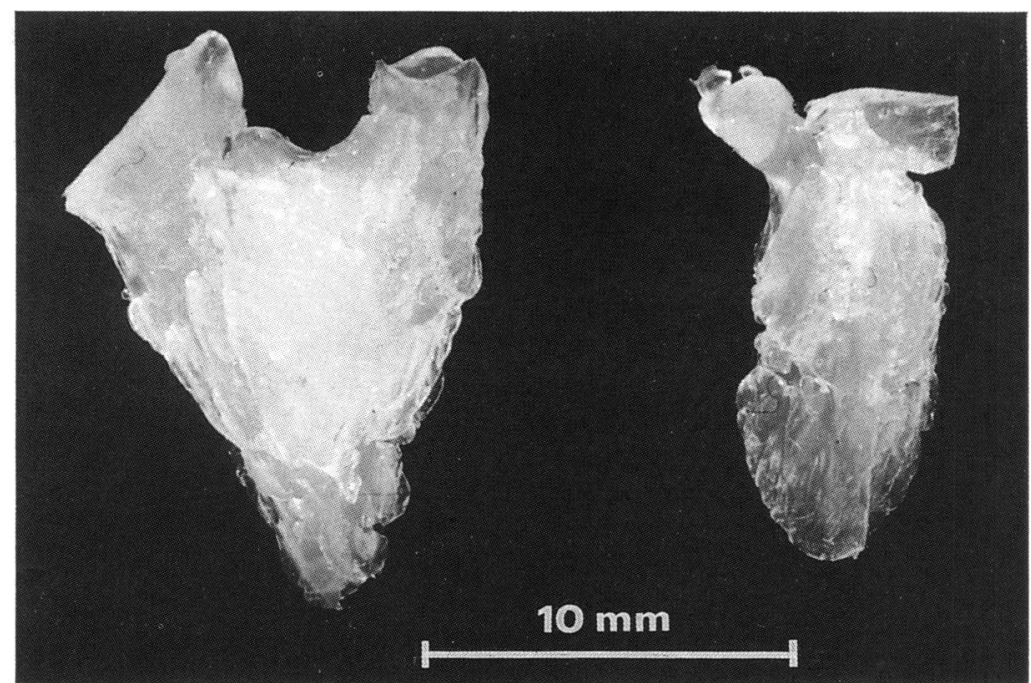

Figure 1 Casts of right and left ventricular cavities are photographed with their septal surface against the background. Morphology, shape, and size of ventricles can be compared in this case of coarctation of the aorta in a 19 week fetus.

catalysts have been used in postmortem material to study examples of congenital heart disease and to assist in the interpretation of specimens after surgical reconstruction of cardiac malformation. ${ }^{6}$ We adapted this method for use in the analysis of cardiac abnormalities in the fetus.

\section{Patients and methods}

Three specimens of normal fetal hearts from spontaneous abortions were casted to evaluate the cardiac anatomy. Of a series of fetal specimens obtained after termination of pregnancy for congenital heart disease, 32 were selected for silicone cast injection. The gestational age of the cases studied was from 18 to 26 weeks with a mean of 20.6 weeks. Both fresh and formalin fixed specimens were used. Anatomical dissection was carried out to expose the intrathoracic organs in situ. External appraisal of the anatomy was performed. In

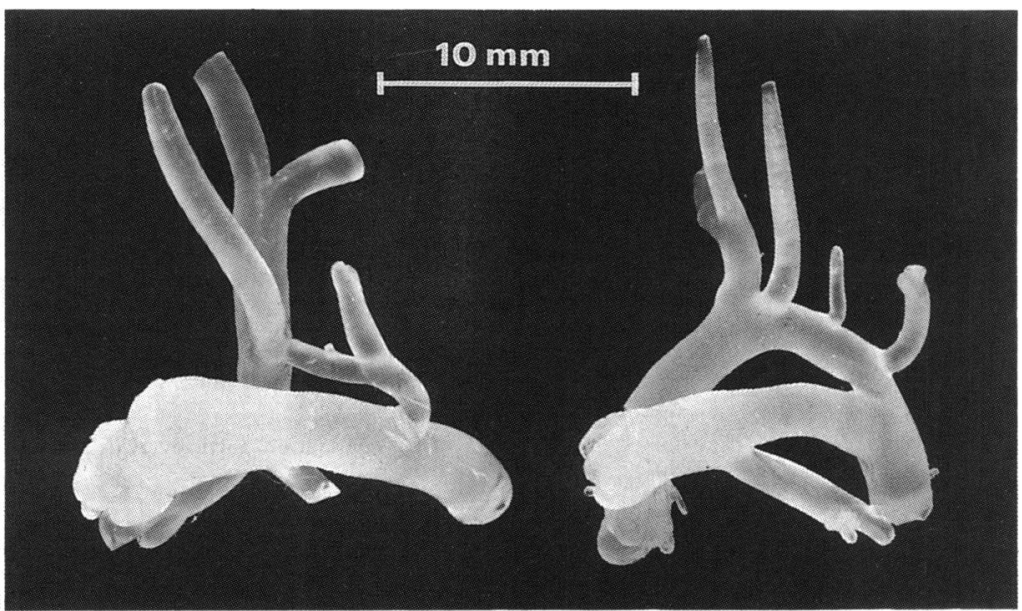

Figure 2 Arch anatomy can be compared in two fetuses of 19 weeks gestation. Coarctation of the aorta is shown on the left and a normal arch on the right. The transverse arch and arterial duct are of similar size normally with the pulmonary root slightly larger than the aorta at its origin. In this example of coarctation, the transverse arch is less than half the normal width and there is an acute angle between the isthmus and descending aorta at the site of a coarctation shelf. The origin of the pulmonary artery is nearly twice the size of the ascending aorta. cases where it appeared possible that casting would improve the definition of cardiovascular anatomy, a small incision was made downstream to the anatomical structure to be investigated. The incision was in the left atrium in 13 , the aorta in 12 , the pulmonary trunk in five, and other sites in five. Blood clots were washed out of the cavities and vessels with a water filled syringe. The casting material used was a commercially available silicone rubber sealant (Dow Corning house seal). This compound was found to be self setting over a period of one day in formalin. The material was injected gently from a syringe through a small pipette positioned in the incision. Distension of the chambers and vessels was observed as the infusion took place. In some cases, the casting material was massaged along the vessel under study until it reached the descending aorta. It was important to ensure that vessels and chambers of interest were filled with the material to avoid artefacts. The heart and lungs were removed from the thorax 24 hours after the cast injection. The cardiac anatomy was then dissected according to a standard method. ${ }^{7}$ The cast was removed carefully from the atrium, ventricle, and great artery in progressive order, preserving the integrity of valvar tissue, papillary muscles, and small vessels.

\section{Results}

Figures 1-6 illustrate some examples of the specimens that were obtained from the 35 cases. The table lists the echocardiographic diagnosis and the additional findings in the cases of congenital heart disease studied. The case illustrated in figs 1 and 2 left is one of coarctation of the aorta. The echocardiographic findings at 19 weeks of gestation were the right ventricle larger than the left; the pulmonary artery larger than the aorta; and distal arch hypoplasia in transverse sections. Figure 1 compares the casts obtained from each ventricular chamber, clearly showing the right ventricle to be nearly twice the size of the left. Figure 2 right shows a normal aorta, aortic arch, pulmonary artery, and duct in a 19 week specimen. Normally the pulmonary artery is slightly larger than the aorta but in fig 2 left, this difference is seen to be much greater than normal. The distal arch narrows considerably after the common carotid branch and the isthmus shows an acute angle at the junction between the duct and the descending aorta. The coarctation shelf indents the posterior aspect of the cast at this point. Figures $3 \mathrm{~A}, \mathrm{~B}$, $C$, and $D$ show some of the varieties of coarctation which can occur. In the case illustrated in figure $3 \mathrm{~A}$, there is only a discrete shelf opposite the junction of the duct with the aorta. Figure 3B illustrates that there is isthmal hypoplasia in a fetus with a subaortic ventricular septal defect and a hypoplastic left ventricular cavity. Figure 3C shows more diffuse transverse arch hypoplasia extending from before the brachiocephalic artery to the isthmus. Figure 3D shows tubular hypoplasia from the aortic valve to the isthmus before the coarctation shelf. Figure 4 shows a whole cardiac cast with mitral atresia 


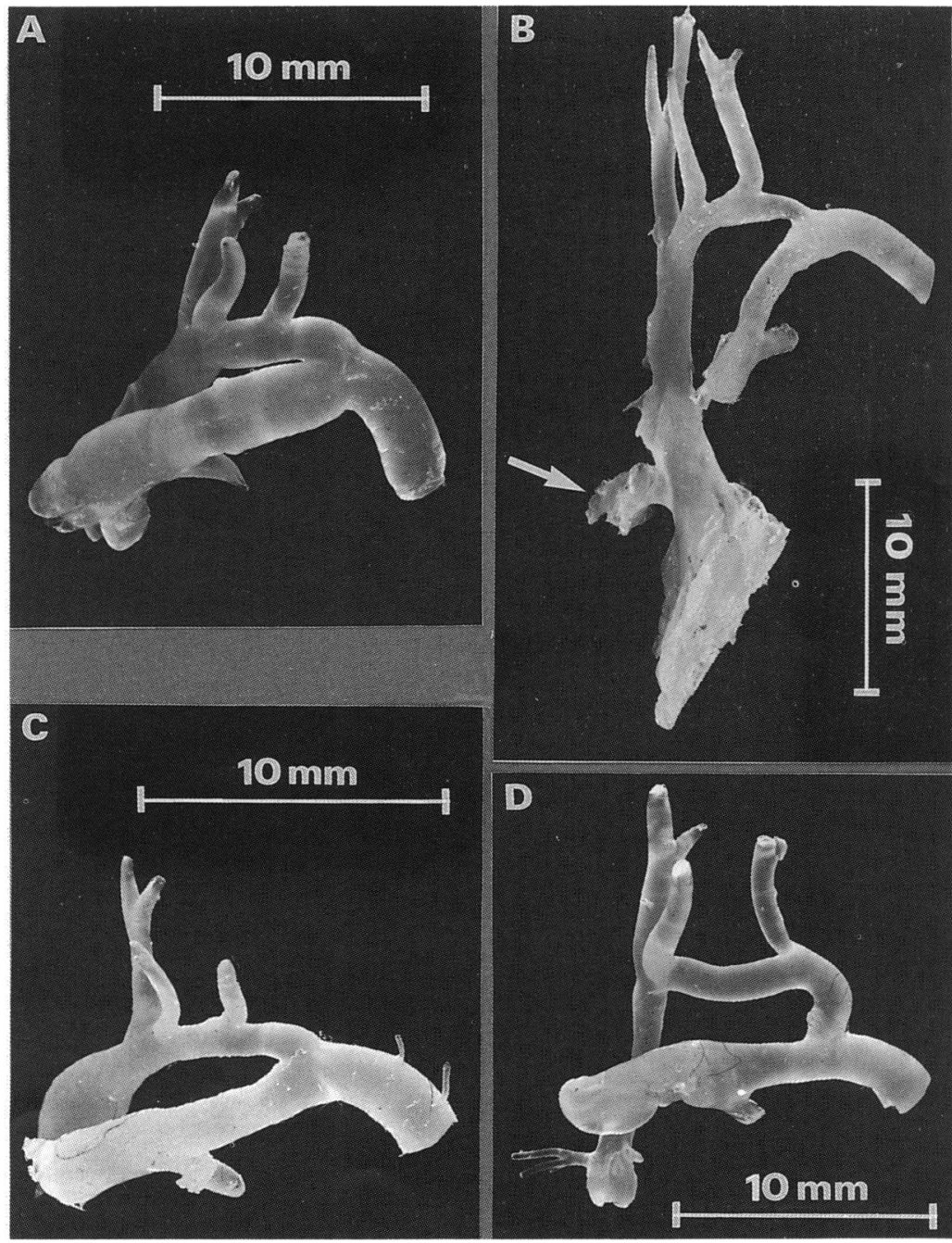

Figure 3 This series shows variation in arch abnormalities. (3A) Arch structures are of comparable sizes but a discrete coarctation shelf is seen indenting the cast in the juxtaductal position. (3B) A cast from the mitral valve to the descending aorta is shown. The left ventricle was hypoplastic although this is difficult to appreciate without the right for comparison. Cast material has crossed a suboartic ventricular septal defect (arrow). Hypoplasia of the arch is limited to the isthmus and there is a mild coarctation lesion. (3C) The arch becomes hypoplastic just before the origin of the brachiocephalic artery and remains so until the junction with the arterial duct. (3D) Tubular hypoplasia of the whole aorta to the junction with the duct is seen, most obvious in the ascending aorta.

Figure 4 Whole cardiac cast from the atria to the descending aorta. The pulmonary artery is divided at its origin and reflected to the right to display the hypoplastic left ventricle. A trabecular and subaortic ventricular septal defect are seen. The aorta arises astride the ventricular septum. The distal arch is hypoplastic and a coarctation lesion is also seen.

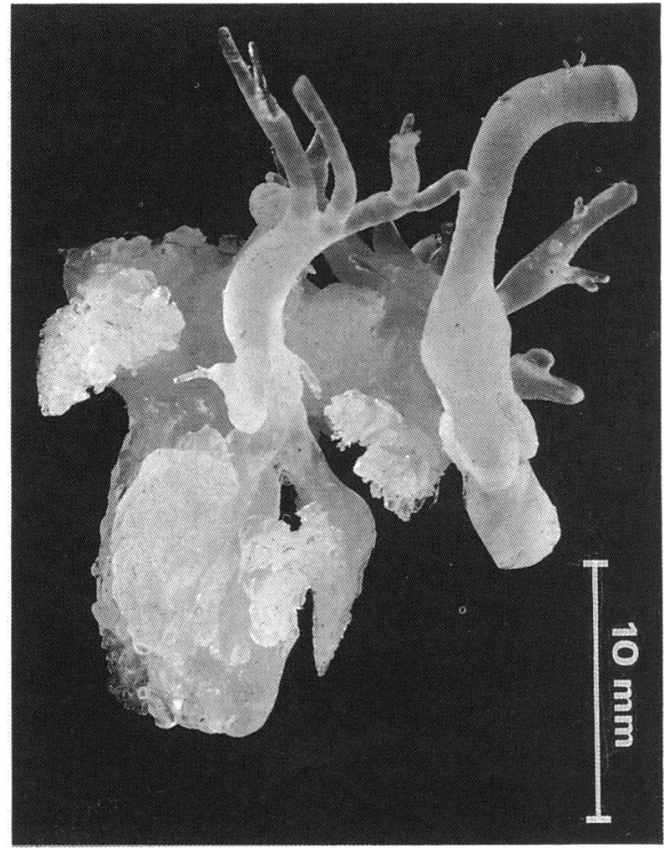

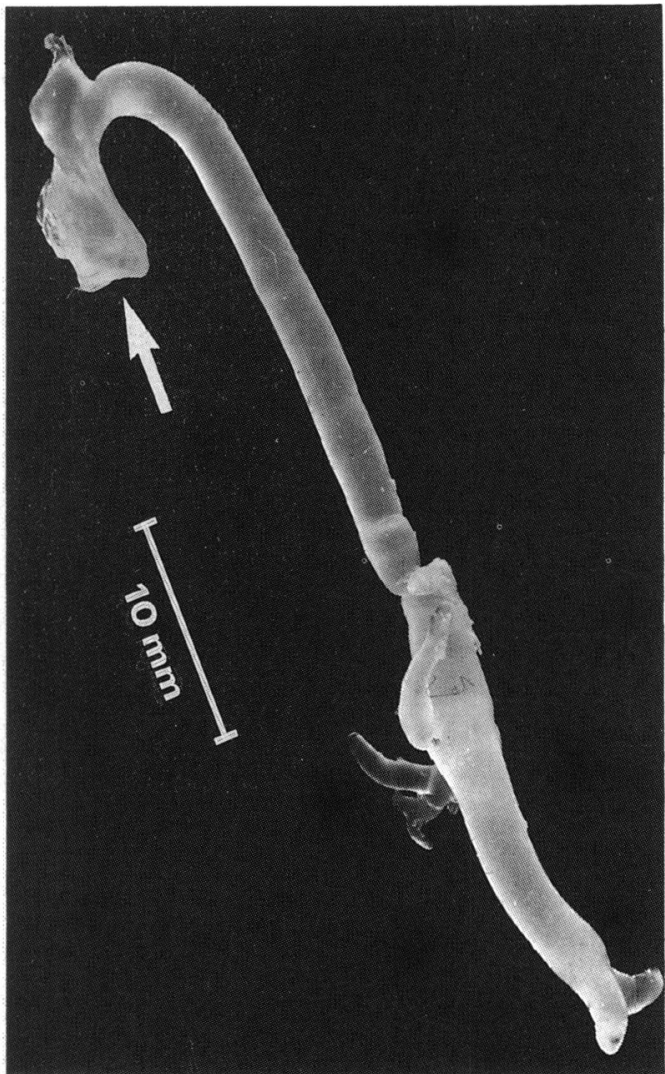

Figure 5 Renal veins are seen joining the venous system below the diaphragm. The azygos vein lay behind the aorta and connected to a left superior vena cava at its junction with the left atrium (arrow).

and left ventricular hypoplasia, and a muscular subaortic ventricular septal defect. The aorta arises astride the ventricular septum and there is transverse arch hypoplasia before a coarctation shelf. A visual understanding of the comparative ventricular sizes can be readily appreciated in the cast specimen. Figure 5 illustrates a case in which there was interruption of the inferior vena cava on echocardiographic examination of the abdominal vessels below the diaphragm in a 18 week fetus. There was a venous structure lying behind the aorta that could be seen on colour flow mapping to join a left sided superior vena cava at its junction with the left atrium. Figure 5 shows the cast of an azygos system obtained from this fetus, which correlated completely with the prenatal findings. This delicate vessel is often damaged or even destroyed on removing the thoracic viscera from the body. Figure 6 illustrates a case in which there was a hypertrophied right ventricle with a small cavity found on echocardiography at 24 weeks' gestation. The pulmonary outflow tract was atretic from the infundibulum to the pulmonary valve. The cast injection confirmed those findings but also showed a large fistulous connection between the left anterior descending coronary artery and the trabecular portion of the right ventricular cavity. Measurement of the casted structures and chambers showed a close correlation with those obtained by echocardiography in all cases where measurements were made. 
Figure 6 Small right ventricular cavity from the tricuspid valve to the atretic infundibulum (arrow). Left circumflex and anterior descending coronary arteries are seen arising from the aortic root. A sinusoidal connection between the midtrabecular portion of the right ventricle and the left anterior descending coronary artery is seen.

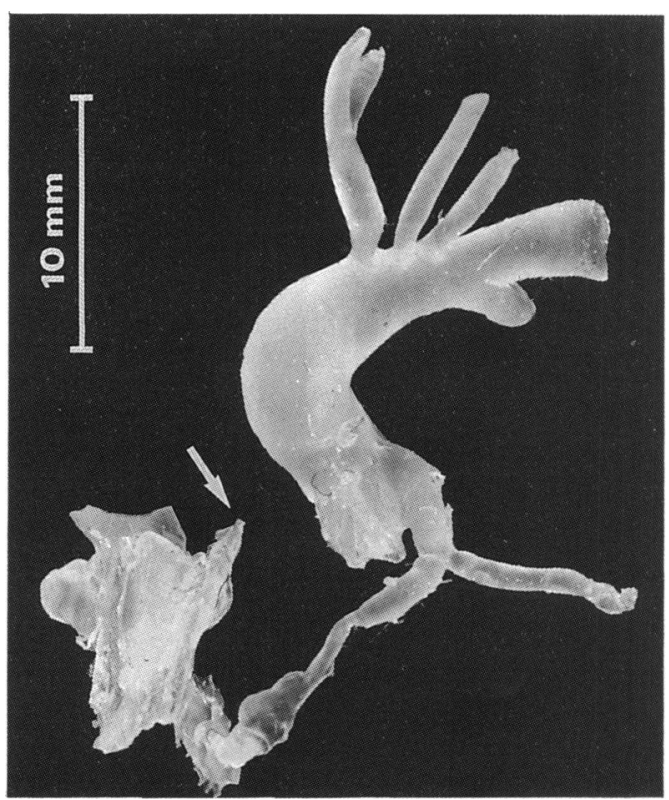

Discussion

This newly described technique proved particularly useful in the correlation of fetal echocardiography with pathological specimens in our series. The technique is simple and can be applied routinely. It has the advantage of maintaining the integrity of the specimen itself and producing a three dimensional picture of the cardiac cavities. Despite the small size of the fetal specimens, we achieved exquisite detail of structures less than one millimetre in size. We found that casts enhanced the interpretation of morphology, displayed paths of flow, and avoided the distortion that can occur during a standard pathological demonstration. Cast models were particularly useful as a teaching aid. Defects could be detailed in their entirety by the cast. The frequency of aortic arch lesions as underlying or associated findings in the hypoplastic left heart syndrome was demonstrated in cast material and the extent of hypoplasia of the left ventricle and arch was defined.

Echocardiographic diagnosis could be confirmed and in some cases extended-for example, in the case of the coronary artery sinusoid. More faithful representation of the cardiovascular system was obtained where casting was performed in situ; this is a practical technique in the fetal specimen. We are currently assessing the possibility of using the cast model to examine in closer detail the characteristics of valve morphology in cases of fetal valvar stenoses.

In summary, casting has proved a useful addition to the morphological analysis of fetal heart specimens. It is easy and practical to use. It provides a three dimensional model that is ideal as an aid to understanding the nature and extent of congenital heart malformations, particularly in the early fetus.

AC and LDA are supported by the British Heart Foundation

1 Lange LW, Sahn DJ, Allen HD, Goldberg SJ, Anderson C Giles $H$. Qualitative real-time cross-sectional echocardiographic imaging of the human fetus during the second half of pregnancy. Circulation 1980;62:799-806.

2 Kleinman CS, Hobbins JC, Jaffe CC, et al. Echocardiographic studies of the human fetus: prenatal diagnosis of congenital heart disease and cardiac dysrhythmias. Pediatrics 1980;65:1059-67.

3 Allan LD, Tynan MJ, Campbell S, Wilkinson J, Anderson $\mathrm{RH}$. Echocardiographic and anatomical correlates in the fetus. Br Heart J 1980;44:444-51.

4 Allan LD, Crawford DC, Anderson RH, Tynan MJ. Echocardiographic and anatomical correlations in fetal congenital heart disease. Br Heart J 1984;52:542-8.

5 Thompsett DH. Anatomical techniques. 2nd ed. London: Churchill, 1970:153-6

6 Kilner PJ, Ho SY, Anderson RH. Cardiovascular cavities cast in silicone rubber as an adjunct to postmortem examination of the heart. Int J Cardiol 1988;22:99-107.

7 Devine WA, Debich DE, Anderson RH. Dissection of congenitally malformed hearts, with comments on the value of sequential segmental analysis. Pediatr Pathol 1991;11:235-59. 